\title{
Molecular Dynamics Study of the Tensile Deformation on Aluminum nanorod
}

\author{
Yongli Liu, ${ }^{1, a}$, Dongkai Shao ${ }^{2, b}$, Yuntao Zeng ${ }^{3, c}$, Weike Wang ${ }^{4, d}$, Mingqi Lu ${ }^{5, e}$ \\ ${ }^{1}$ Institute of Materials Physics and Chemistry, College of Material Science and Engineering, \\ Northeastern University, \\ Shenyang 110819, China. \\ ${ }^{2}$ Institute of Materials Physics and Chemistry, College of Material Science and Engineering, \\ Northeastern University, \\ Shenyang 110819, China \\ ${ }^{3}$ Institute of Materials Physics and Chemistry, College of Material Science and Engineering, \\ Northeastern University, \\ Shenyang 110819, China. \\ ${ }^{4}$ Institute of Materials Physics and Chemistry, College of Material Science and Engineering, \\ Northeastern University, \\ Shenyang 110819, China \\ ${ }^{5}$ Institute of Materials Physics and Chemistry, College of Material Science and Engineering, \\ Northeastern University, \\ Shenyang 110819, China \\ aylliu@imp.neu.edu.cn, bdongk.shao@gmail.com, 'cstickzyt@163.com, ${ }^{d} 1265456599 @ q q . c o m$, \\ elumq9418@163.com
}

Keywords: Molecular dynamics, Tensile deformation, Nanorod.

Abstract. To study the deformation behavior of the nanorods and further explore the deformation difference among nanorods, nanowires and bulk materials, the uniaxial tension of aluminum nanorod under different stretching rate was conducted by the molecular dynamics method. The effect of stretching rate on the tensile deformation of nanorod was investigated by considering the variation of the energy, engineering stress, yield stress, Youngs modulus changes with the strain. Further the deformation mechanism was discussed based on the study of the snapshots of the microstructure transformaiton at different stages. It was found that, when the stretching rate is under $9.26 \times 10^{9} \mathrm{~s}^{-1}$, the deformation behavior of the aluminum nanorod was similar with that of aluminum nanowire and crystal at room temperature, and presents one necking fracture mechanism. We can obtain high yield strength and yield strain. However, when the strain rate exceeding $9.26 \times 10^{9} \mathrm{~s}^{-1}$, the nanorod fractured near the boundaries and presents two necking mechanism, which is greatly different with that of the nano-wire or bulk materials.

\section{Introduction}

In the past decade, nanomaterials have attracted a great deal of interests due to its unique mechanical, electronic, optical and magnetic properties, opening up a broad view of applications [1-2]. Practical need in micro electron mechanical systems (MEMS) and nano electron mechanical systems (NEMS) requires further investigation and understanding of the deformation properties of the nanomaterials urgently. Aluminum alloys possess the advantages such as high strength, good formability and weight saving. It is necessary for us to study the joints, deformation and the welding properties of the nano-scaled aluminum, magnesium, and $\mathrm{Al}-\mathrm{Mg}$ alloys both from the experimental and theoretical aspects. 
Characterization of mechanical properties and microstructure evolution during deformation is an important area in material science. Some testing and measuring techniques like nano-indentation tests with extremely light loads has already been developed in the mid-1970s [3]. In recent years, new test methods with Scanning Probe Microscope (SPM) were used to characterize nanomaterial's Young's modulus [4-5]. However, the mechanisms exploration and microstructure characterization of nanomaterial is still difficult due to the complexity of equipments and the uncertainty of the samples. So, the simulation methods, especially molecular dynamics (MD) method, were often adopted as an optimum approaches in exploring mechanical property of nanomaterials [7-14]. Some of the simulations were conducted on single crystal to study the shearing deformation [7], the void effect [8], the softening behavior [9], the stretching deformation [10], and the effects of different model sizes, boundary conditions, crystal orientations and load speeds on tensile deformation [11]. The other simulations were conducted on the nanowires to study the abnormal stress drop at the yield point [12], the uniaxial tensile deformation [13] and the breaking process of the gold nanowires [14]. The previous study of nanocrystal and nanowires had presented some special properties comparing with the bulk crystals, such as the absence of the hardness, the one necking mechanism, a drop of the stress at the yield point [15], little dependence of the Youngs modulus on the loading rate, and possesses higher yield stress. For aluminum and its oxides, though the nanorod was successfully fabricated with different methods in the laboratories [16-18], the corresponding mechanical and microstructure study is extremely limited. To achieve a deep understanding of the jointing and deformation properties of $\mathrm{Al}$ and other metals, it is necessary to study the correlated properties of the nanorod. The present work will study the effects of stretching rate on the uniaxial tensile deformation and the structural evolution at room temperature $(300 \mathrm{~K})$ by using molecular dynamics methods. The rest of the paper was organized as follows: In Section 2, the detailed MD simulation techniques and the deformation conditions and models were introduced. In Section 3, the relationship between strain rate and fracture behavior were demonstrated and discussed by analyzing the average energy, engineering stress and strain, and the snapshots of deformation process at different stages. The summary and conclusions were given in Section 4.

\section{Method and model}

The modified FS potentials for Al-Mg alloys developed by Mendelev et. al. [19] was adopted In the present work. Mendelev's potential fitted the ab initio data and Al-rich part of the measured solid-liquid phase diagram fairly well, and had obtained creditable simulation results [20-23]. The total energy of the $\mathrm{N}$ particles' system takes the following form

$$
\begin{gathered}
U=\sum_{i=1}^{N-1} \sum_{j=i+1}^{N-1} \varphi_{t_{i}} \varphi_{t_{j}}\left(r_{i j}\right)+\sum_{i=1}^{N} \varphi_{t_{i}}\left(\rho_{i}\right) \\
\rho_{i}=\sum_{j} \psi_{t_{i} t_{j}}\left(r_{i j}\right)
\end{gathered}
$$

Where $t_{i(j)}$ is the elemental type of atom $i(j) \mathrm{N}$ is the number of atoms in the system, $r_{i j}$ is the distance between atom $i$ and atom $j, \psi_{t_{i} t_{j}}$ is the pair-wise potential, and $\psi_{t_{i}}(r)$ is the embedding energy function. The expression of density function was shown in Equation (2).

The initial geometry of sample was constructed in a box of $30 \mathrm{a}_{0} \times 30 \mathrm{a}_{0} \times 80 \mathrm{a}_{0}\left(\mathrm{a}_{0}\right.$ is the lattice constant of aluminum), where the crystallographic orientations along the $\mathrm{X}, \mathrm{Y}$ and $\mathrm{Z}$ axis were taken as $\left[\begin{array}{lll}1 & 0 & 0\end{array}\right]$, $\left[\begin{array}{lll}0 & 1 & 0\end{array}\right]$ and $\left[\begin{array}{lll}0 & 0 & 1\end{array}\right]$. Then a cuboid aluminum nanorod model was built within this box with 16 unit cells along $\mathrm{X}$ and $\mathrm{Y}$ direction and 32 unit cells along $\mathrm{Z}$ direction. Thus the total volume of the simulated nanorod was $129.6 \AA$ in length and $64.8 \AA$ in width and depth. Along the $Z$ direction, two 'reservoir' regions of 10 atomic planes were fixed in both sides of the model. The atoms in the reservoir region moved together and didn't experience the relative movement during the deformation process. For the non-reservoir regions, the atoms moved under the combination of interatomic action and external force field. The time-step was 0.5 fs $\left(0.5 \times 10^{-10} \mathrm{~s}\right)$. The stretch deformation of Aluminum nanorod was 
conducted on the Large-scale Atomic/Molecular Massively Parallel Simulator (LAMMPS) platform [24].

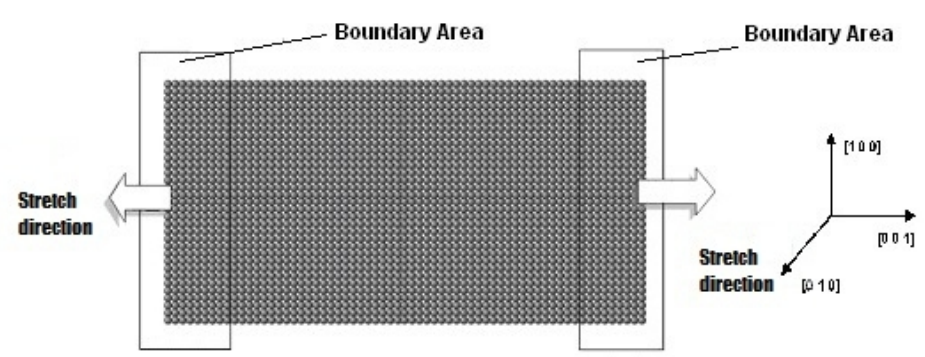

Fig.1 The initial atomic configuration of aluminium nanorod, the stretch deformation was applied along the Z-axis ([001] direction) with constant strain rate.

\section{Results and Discussion}

The simulation of the stretching deformation initiated after a relaxation of 100000 MD time-steps. To investigate the influence of strain rate, different stretching rates ranging from $1.54 \times 10^{9} \mathrm{~s}^{-1}$ to $1.35 \times 10^{10} \mathrm{~s}^{-1}$ were studied. Due to the time scale of molecular dynamics, only short period of time was considered, and thus the strain rate adopted here was very high compared to that in experiment. The effect of stretching rate on the nanorod deformation was initially considered through the variation of the energy, stress, and Young's modulus changes with the strain. Then according to the unusual phenomenon featured by the stress-strain curves, the microstructure snapshots were extracted and demonstrated to reveal the detailed structural evolution vividly.

\section{Effect of stretching rate on the energy, stress and Young's modulus}

The variation of the average energy changes with the strain was shown in Fig.2. It can be found that the average energy initially increased linearly and then commonly meet a stage of $3.325 \mathrm{eV} / \mathrm{atom}$. This value should be related with the cohesive energy $(3.36 \mathrm{eV} /$ atom $[1,10,12])$. Because of the existence of the rod surface, the present energy is a little higher than that of the bulk systems. Since the cohesive energy is the intrinsic properties, all of the present rods show the same value, which is independent of the stretching rate.

When the stretch continues, the average energy increases to the maximum with another tiny stagnation, and then rapidly dropped to a locally steady value, implying the completion of the uniaxial deformation. Note that with the increment of the stretching rate, the strain reasonably enlarged, but the maximum energy doesn't present a monotonous trend. The application of the external strain will distort the lattice from the equilibrium, and the response of internal force will tend to revert the change. Therefore, the stretching process is also the competition between the external force field and the internal stress. The microstructure transformation always happened till the internal resilience meeting its limit. Therefore, the maximum energy can be related with the stretching energy barrier. The maximum energy barrier happens when the tensile rate changes between $7.7 \times 10^{9}-9.24 \times 10^{9} \mathrm{~s}^{-1}$. Therefore, the nanorod stretching within this rate range possesses a better anti-stretching ability than other conditions. 


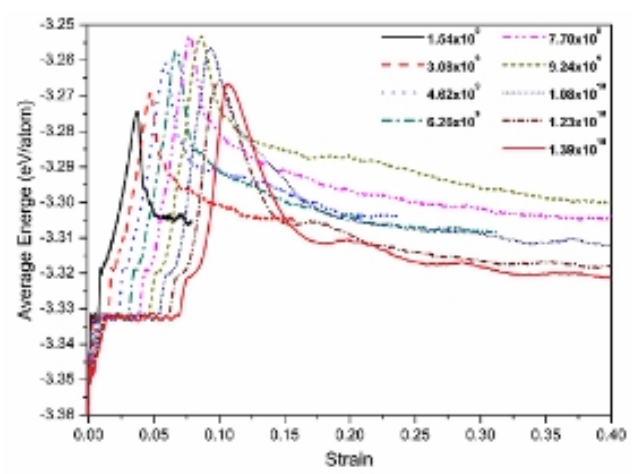

Fig. 2 The energy-strain curves of aluminum nanorod at different stretching rates

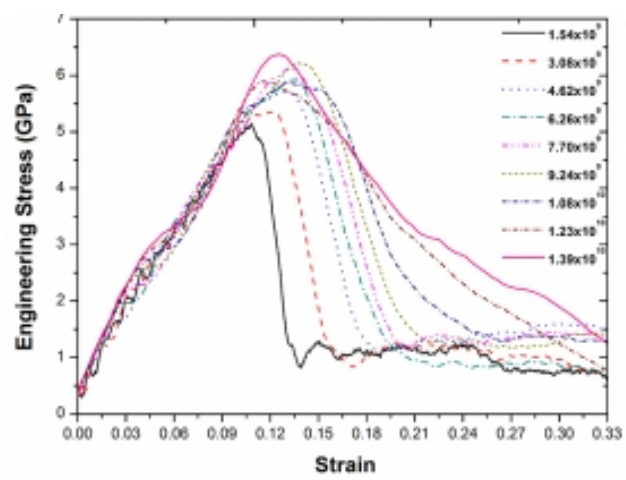

Fig. 3. The stress-strain curves of aluminum nanorod at different stretching rates.

The engineering stress and strain curve with different stretching rates was shown in Fig.3. We see that the initial stage of the stress increases linearly up to $10 \%$ and nearly overlaps regardless of the stretching rates. This indicates that the elastic property of a nanorod is insensitive to stretching rate. This phenomenon proves that Young's modulus is the inherent properties of the aluminum nanorods. This situation was similar with that of the nanowire but differentiated with the nanocrystals [12-15]. The Young's modulus of [ 001 ] direction is about $44.8 \mathrm{GPa}$ at $300 \mathrm{~K}$, which is $65 \%$ of its bulk value (69 GPa) [25]. Compared with previous calculated Young's modulus $(35-65 \mathrm{GPa}[1,26,12])$ of aluminum, present magnitude is acceptable.

The stress typically increases with the increment of the strain at the initial elastic stage, and then drops abruptly when they meet the highest value (Fig.3), indicating the occurrence of the plastic deformation. The following plastic deformation presents the zigzag behavior under low strain rate, that may related with the stick-slip behavior of the atomic planes and the nucleation and slip of the dislocation. This phenomenon agrees with the simulation behavior of the Al nanocrystal [10], Fe nanocrystal [11] and Ni nanowires [13]. However, with the increment of the stretching rate, the zigzag mode disappears, implying the different slide mode of the atomic plane during the deformation process. The difference will be discussed in the following section.

\section{Effect of stretching rates on the yield strength and the engineering strain}

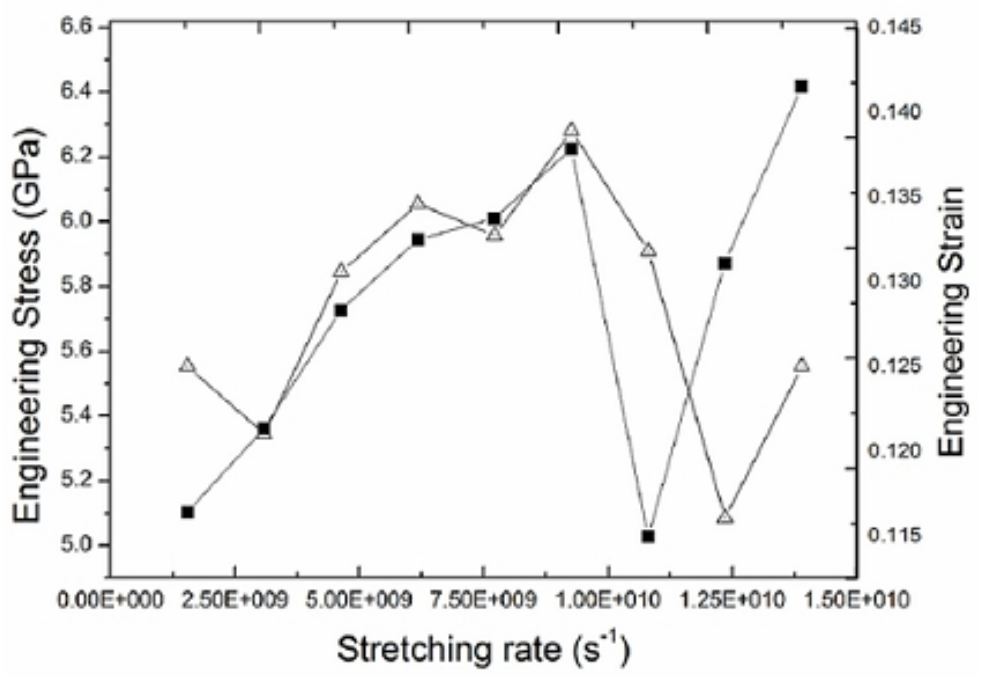

Fig.4. the variation of the engineering stress and strain changes with the stretching rates.

From Fig. 4 we can see that, before the sample completely transforming into the plastic deformation, both the maximum stress and the elastic strain present great changes with different stretching rates. Here we consider the highest stress in the deformation process as the yield strength according to the general simulation rule [27]. Either the yield strength or engineering strain can reveal the working limit in practical application. The relation variation of the yield strength and engineering strain as a function 
of the stretching rate was shown in Fig.3. Further, with the increment of stretching rate, the engineering stress presents two peaks. The engineering strain also presents the similar trend with that of the yield strength with a little delay (Fig.4). According to the variation of engineering stress and strain, we can classify the effect of stretching rate on the nanorod into three deformation regimes, i.e. smaller than $1.54 \times 10^{9} \mathrm{~s}^{-1}$ (regime I), between $1.54 \times 10^{9} \mathrm{~s}^{-1}$ to $9.26 \times 10^{9} \mathrm{~s}^{-1}$ (regime II), and larger than $9.26 \times 10^{9} \mathrm{~s}^{-1}$ (regime III), the corresponding deformation modes was noted as type I, II or III deformation. For type I deformation, the lowest yield strength and engineering strain is obtained. This phenomenon is in accordance with the phenomenological attributes of plasticity observed in macro-scale experiments that the lower strain rates results in the lower yield stress. For type II deformation, both the yield strength and the engineering strain get increased with the increment of the stretching rate. The maximum yield stress and engineering strain was obtained when the stretching rate varies between $7.7 \times 10^{9}-9.24 \times 10^{9} \mathrm{~s}^{-1}$. This result is in accordance with the information implied in Fig.2. However, for type III deformation, with the increment of the stretching rate, both the yield strength and the engineering strain decreases greatly with the previous mode, implying that the extremely large stretching rate may deteriorates the deformation ability and should be carefully avoided.

\section{Microstructure evolution and the necking mechanism}

To explore the inherent fracture mechanisms of these deformations, the microstructure evolution with the stretching rate of $1.54 \times 10^{9} \mathrm{~s}^{-1}, 6.17 \times 10^{9} \mathrm{~s}^{-1}$ and $9.26 \times 10^{9} \mathrm{~s}^{-1}$ were typically selected to study the microstructure transformation mechanism of the aluminum nanorod during the I, II and III deformation stages.

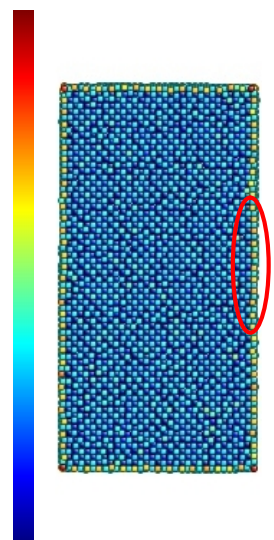

(a) $\mathrm{t}=10000 \mathrm{t}_{0}$
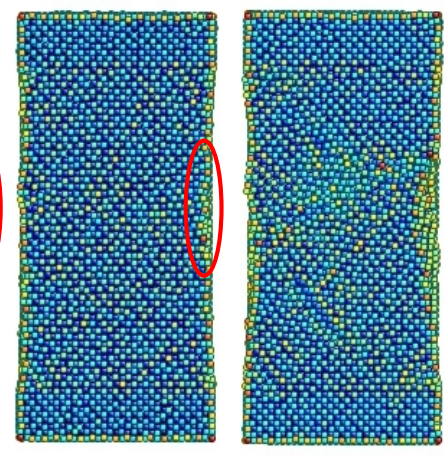

(b) $\mathrm{t}=43000 \mathrm{t}_{0}$

(c) $\mathrm{t}=505000 \mathrm{t}_{0}$

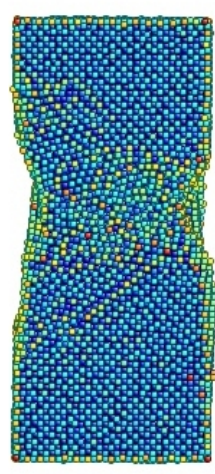

(d) $\mathrm{t}=58000 \mathrm{t}_{0}$

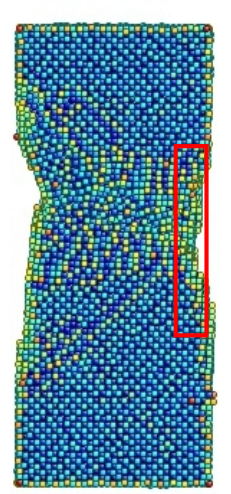

(e) $t=73500 t_{0}$
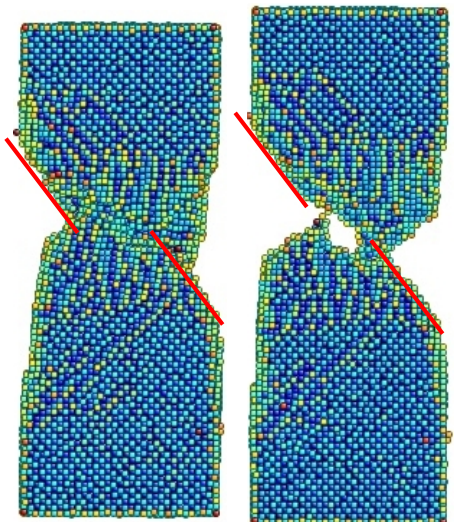

(f) $\mathrm{t}=99000 \mathrm{t}_{0} \quad(\mathrm{~g}) \mathrm{t}=124500 \mathrm{t}_{0}$

Fig.5 The snapshots of aluminum nanorod along $\mathrm{z}$ direction at $300 \mathrm{~K}$ with stain rate of $1.54 \times 10^{9} \mathrm{~s}^{-1}$ at different time-steps. The red (blue) edge of the energy scale bar notes the atoms with high (low) energy.

Fig. 5 presented the snapshots of the microstructure evolution of the deformation with the stretching rate of $1.54 \times 10^{9} \mathrm{~s}^{-1}$. The shape of the deformation model almost unchanged after relaxation (Fig.5 (a)). The situation continues until the nanorod meets the highest strength of $5.1 \mathrm{GPa}$ with the strain of 12.5 $\%$ (Fig.5 (b)). The plastic deformation initiates with relative micro rotating behavior of the low and up parts of the rod (Fig.5 (c)). Continued strain induces the obvious microstructure transformation, such as the micro fracture of the central atomic planes along the direction of $55^{\circ}$ deviating the [ 100$]$ axis, i.e. [111] direction. Also, the reconstructed atomic stacking in the central region along the stretching direction is observed (Fig.5 (d), (e) and (f)). We can see that the atomic layer just slipped along the [111] direction, and several layered dislocation stages occur in the fracture surface. The stretching necking occurs corresponding to the grain gliding direction and quickly fractured when the nanorod is fairly thin (Fig.5 (f) and (g)). The microstructure transformation typically reflects the stick-slip behavior in the metal, and also in accordance with the zigzag change mode in Fig.3. In sum, the mechanism of type I deformation at the low strain rate is similar with that of the behaviors of other simulated nanocrystals and nanowires. 

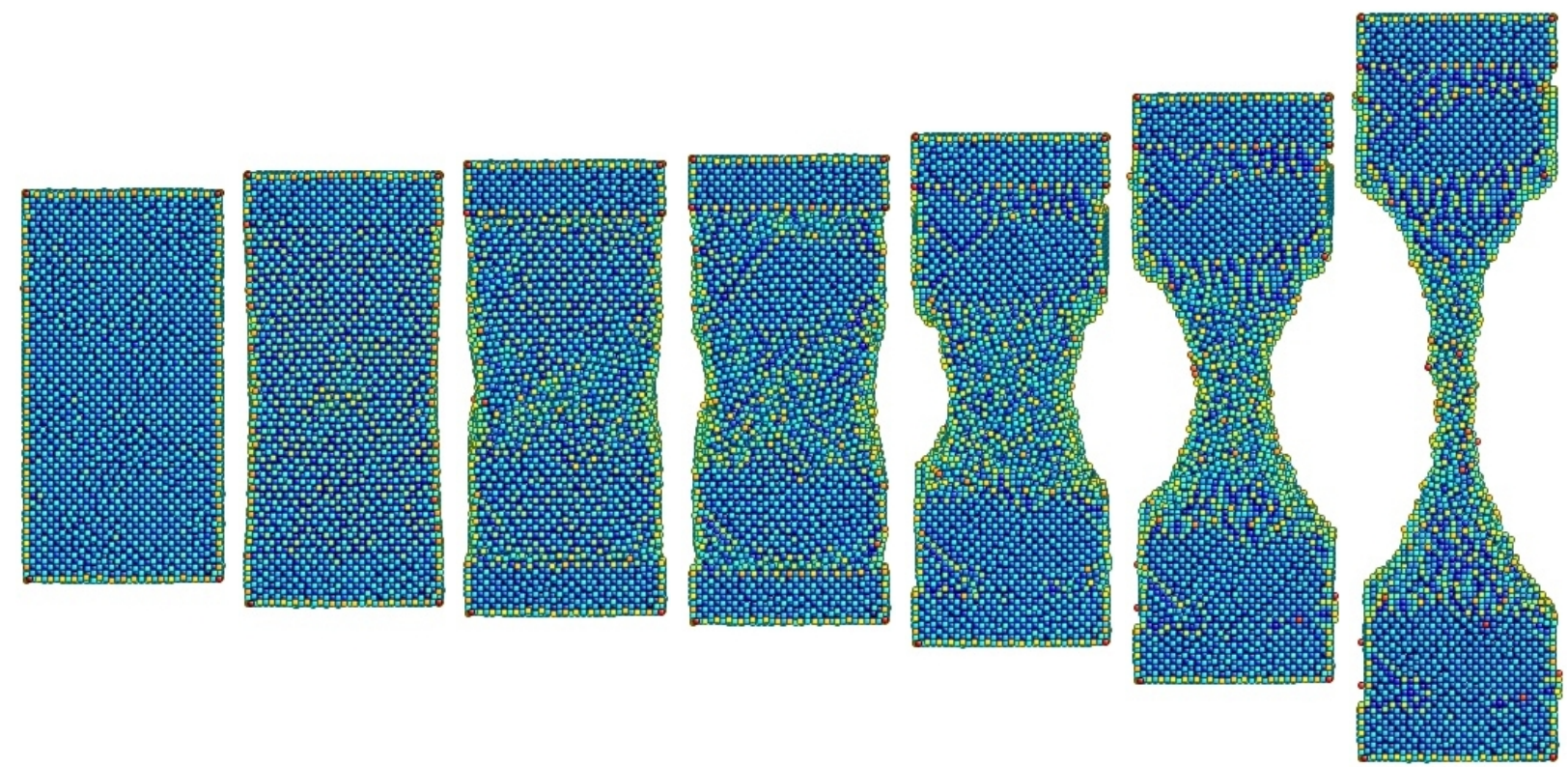

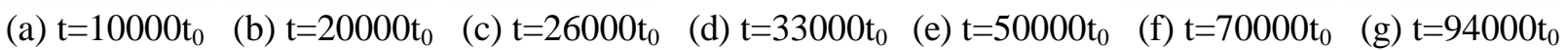
Fig. 6 The snapshots of aluminum nanorod along $\mathrm{z}$ direction at $300 \mathrm{~K}$ with $6.17 \times 10^{9} \mathrm{~s}^{-1}$ at different time-steps. The red (blue) edge of the energy scale bar notes the atoms with high (low) energy.
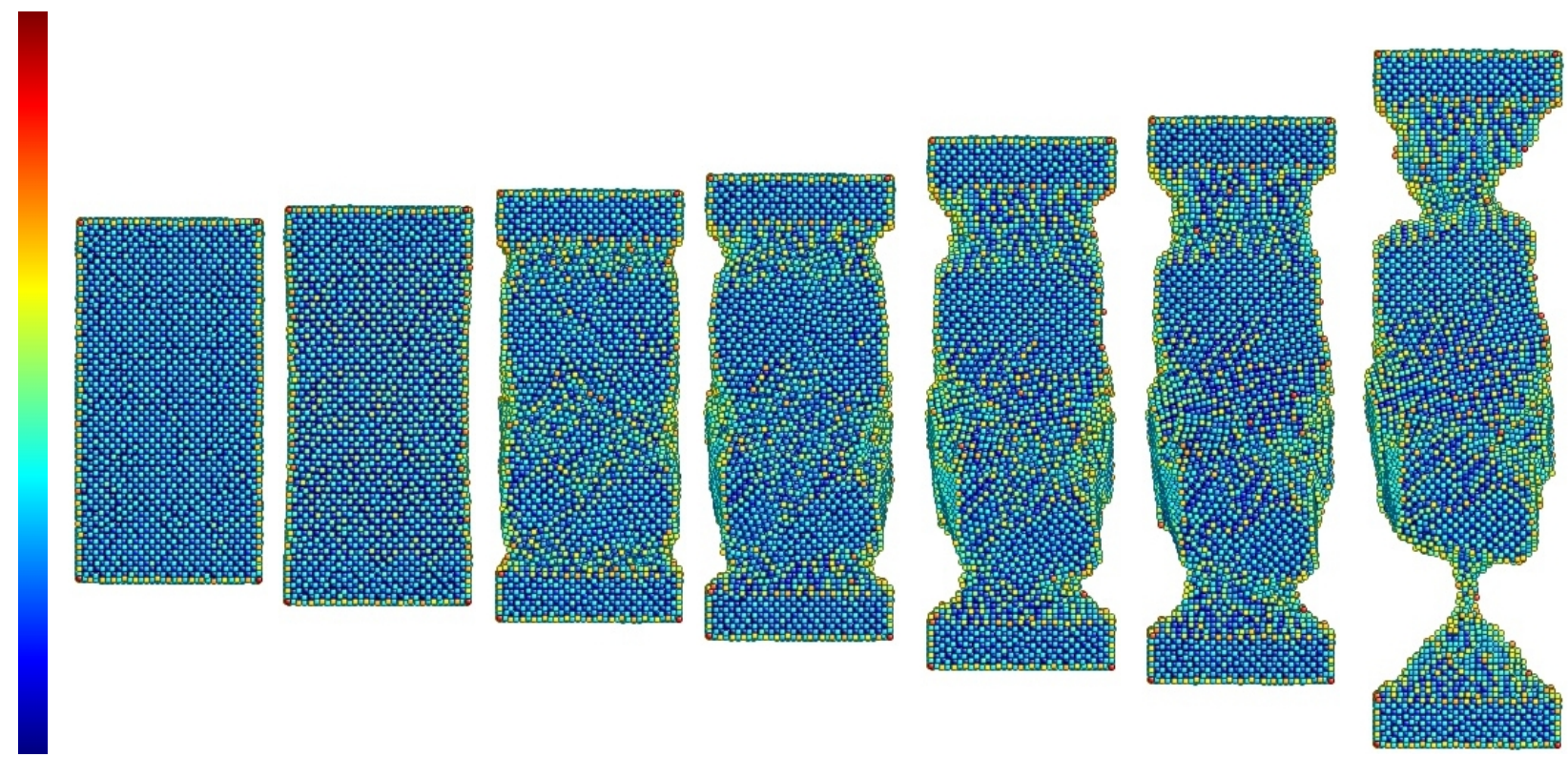

(a) $\mathrm{t}=10000 \mathrm{t}_{0}$ (b) $\mathrm{t}=15000 \mathrm{t}_{0}$

(c) $\mathrm{t}=20000 \mathrm{t}_{0}$

(d) $\mathrm{t}=25000 \mathrm{t}_{0}$

(e) $\mathrm{t}=35000 \mathrm{t}_{0}$

(f) $\mathrm{t}=40000 \mathrm{t}_{0}$

(g) $\mathrm{t}=59500 \mathrm{t}_{0}$

Fig.7 The snapshots of aluminum nanorod along $\mathrm{z}$ direction at $300 \mathrm{~K}$ with stretching rate of $9.26 \times 10^{9} \mathrm{~s}^{-1}$ at different time-steps. The red (blue) edge of the energy scale bar notes the atoms with high (low) energy.

Fig. 6 presented the snapshots of the microstructure during stretching process with the rate of $6.17 \times 10^{9} \mathrm{~s}^{-1}$. The sample meets the highest strength $(5.75 \mathrm{GPa})$ at the strain of $13.5 \%$ (Fig.6 (a) and (b)). The microrotation and fracture at central region are observed (Fig.6 (d), (e)). More than one stages are left in the initial fractured region, which indicated that the plastic deformation imitated with multi slipping of the dislocations on the (111) and (100) planes. With the continuous of the strain field, the flow stress comes into the mainly induction, and the fracture section departed every one atomic 
plane, showing a smooth atomic necking mechanism (Fig.6 (f)-(g)). Less reconstruction of the atomic stacking along the $\left[\begin{array}{lll}0 & 0 & 1\end{array}\right]$ direction are observed because of the high stretching rate than that of the type I deformation.

Compared with the one necking fracture mechanism at strain rate of $6.16 \times 10^{9} \mathrm{~s}^{-1}$, the process of aluminum nanorod with strain rate of $9.26 \times 10^{9} \mathrm{~s}^{-1}$ is much different (Fig.7). Instead of shrink in the middle of the sample, two fracture neckings appear at the regions near the reservoir boundaries at the stage of plastic deformation begin (Fig.7 (b)). And these two shrink regions become two neckings when the strain increases (Fig.7 (c)-(d)). Nearly no atomic sliding is observed during the stretching process (Fig.7 (c)-(g)). This result agrees with the disappearance of the zigzag variation in Fig.2, and can be reasonably understood by considering the preferred driven force. For the atoms in the non-reservoir regions, besides the heat movement correlated with the Boltzmann distribution, its action mainly decided by the surrounded reverse atomic interaction from both sides. Therefore, generally the central section is one typical flimsy region. While for the atoms near the boundary region, it will endure both the external force and the surrounded atomic interaction, thus the section near the boundary is another flimsy region. In conclusion, when the external stretching rate is quite small, the reverse interaction governs deformation process (one necking mechanism, Fig. 5 and 6), and the central section should be the key region to be strengthen; while when the velocity field get big enough, the displacement of the atoms near the boundary become the preferable factor, and the nanorod fractured with two necking mechanism (Fig.7). Generally, the addition of other alloy element may be the effective way to strengthen the alloy. This situation can not been found in nanowire because of the nonexistent boundary. However, we should noticed that $9.26 \times 10^{9} \mathrm{~s}^{-1}$ is just a computational critical strain rate, the corresponding value in practice may be fairly small, but stretching rate still correlates with two different deformation mechanisms. The important implication is that, when the stretching strain is below the critical value, the model can endure large strain and long deformation process with the continue increment of the engineering stress; when the strain rate exceeds the critical value, the sample may possess the leaped stress and quick fracture.

\section{Conclusions}

In this paper, the molecular dynamics methods were adopted to study the effect of the stretching rates on the deformation behavior of aluminium nanorod. It was found that, as most material in nanoscale, aluminum nanorod has extremely high yield strength at room temperature and stretching rate has little effect on the Young's modulus $(44.8 \mathrm{GPa})$ of aluminum nanorod. When the stretching rate is under $9.26 \times 10^{9} \mathrm{~s}^{-1}$, we can obtain high yield strength and yield strain, and aluminum nanorod presents one necking fracture mechanism. However, when the strain rate exceeds $9.26 \times 10^{9} \mathrm{~s}^{-1}$, the nanorod fractures near the boundaries and presents two necking mechanism, and possesses the leaped stress and quick fracture. This kind mechanism is different from the behavior of the manowire or bulk materials. The present results shed some light on the understanding of the stretching properties of the nanorods in the MEMS and NEMS systems.

\section{Acknowledgments}

This research was financially supported by the Fundamental Research Funds for the Central Universities (No. L1502038) and the National Natural Science Foundation of China (No. 51172040 and 51371177). This work was conducted in Shenyang Supercomputer Center at the Institute of Metals Research.

\section{References}

[1]. H. Gleiter: Prog. Mater Sci. Vol.33 (1989), p. 223

[2]. H. Gleiter: Acta Mater. Vol. 48 (2000), p. 1 
[3]. W. H. Chi, D. C. Zhong , G. J. Wang: J Microelectromech S. Vol. 19 (2010), p. 849

[4]. Z. F. Zhu: J Mater Sci. Vol. 45 (2009), p. 46

[5]. Y. Kim: Mater Lett. Vol. 63, (2009), p.1065

[6]. L. Yuan, D. B. Shan et al: J. Mater. Process. Technol. Vol. 184 (2007), p.1

[7]. Y. H. Wen, Z. Z. Zhu, R. Z. Zhu: Comp Mater Sci. Vol. 41 (2008), p.553

[8]. J. B. Pethica, R. Hutchings, W. C. Oliver: Philos Mag A. Vol. 48 (1983), p. 593

[9]. R. Arenal, M. S. Wang, Z. Xu, A. Loiseau, D. Golberg: Nanotechnology. Vol. 22 (2011), p. 265704

[10]. J. J. Roa, G. Oncins, F. T. Dias, V. N. Vieira, J. Schaf, M. Segarra: Physica C. Vol.471 (2011), p.544

[11]. T. J. Young, M. J. Monclus, T. L. Burnett, W. R. Broughton, S. L. Ogin, P. A. Smith: Meas Sci Technol. Vol. 22 (2011), p. 125703

[12]. Y. Yuan, T. Sun, J. J. Zhang, Y. D. Yan: Appl Surf Sci. Vol. 257 (2011), p.7140

[13]. Z. Dirk: Comp. Mater. Sci. Vol. 45 (2009), p. 845

[14]. R. Komanduri, N. Chandrasekaran, L. M. Raff: Int. J. Mech. Sci. Vol. 43 (2001), p. 2237

[15]. E. Z. D. Silva: Phys. Rev. Lett.Vol. 87 (2001), p. 256102

[17]. Y. C. Chen, K. Nakata: Scripta Mater. Vol. 58 (2008), p. 433

[18]. M. I. Mendelev: Philos. Mag. Vol. 89 (2009), p. 3269

[19]. L. Proville: Phys. Rev. B Vol. 82 (2010), p. 054115

[20]. C. C. Wang: J Allloy Compd.Vol. 509 (2011), p. 10222

[21]. J. J. Hoyt, A. A. Potter: Metall. Mater. Trans. A. Vol.43(2012), p. 3972

[22]. L. Shen, G. Proust, G. Ranzi: IOP conference Series-Materials Science and Engineering. Vol. 10 (2010), p. 012177

[23]. W. D. Callister, D. G. Rethwisch : Materials Science and Engineering: An introduction, 9th edition, John Wiley / Sons. Inc, NY (2007)

[24]. S. J. Plimpton: J. Comput. Phys. Vol. 117 (1995), p.1

[25]. J. Schiotz, F. D. D. Tolla, Jacobsen, K. W: Nature. Vol. 391 (1998), p. 564

[26]. R.W.Hertzberg: Deformation and fracture mecahnics of engineering materials, 4th edition, John Wiley / Sons.Inc, NY (1996)

[27]. L. Pastor-Abia,M. J. Caturla, E. SanFabián, G. Chiappe, E. Louis: Phys rev B. Vol. 83 (2011), p. 165441 